\title{
A HYPERBOLIC GCM LIE ALGEBRA AND THE FIBONACCI NUMBERS ${ }^{1}$
}

\author{
ALEX JAY FEINGOLD
}

\begin{abstract}
The Fibonacci numbers are found to be involved with the WeylMacdonald-Kac denominator formula for a certain rank 2 Generalized Cartan Matrix Lie algebra.
\end{abstract}

Introduction. Since their independent introduction by Kac [2], [3] and Moody [8], [9] there has been increasing interest in the Generalized Cartan Matrix (GCM) Lie algebras because of their connections with other parts of mathematics (see [4]). For instance, the Macdonald identities [7] were shown to be the "denominator formula" for the Euclidean GCM Lie algebras [3], [10]. We are concerned here with an algebra which falls into the class of algebras called "hyperbolic", whose root systems were studied by Moody in [11]. An exciting connection was recently found by Lepowsky and Moody in [6] between the rank 2 hyperbolic GCM Lie algebras and Hilbert modular surfaces with respect to a real quadratic field. For one of these algebras the quadratic field is $Q(\sqrt{5})$. In this paper we show that the Fibonacci numbers are intimately related to this algebra by using them to express the Weyl-Macdonald-Kac denominator formula (\$2). We do not compute the root multiplicities, but see $\$ 3$.

1. Preliminaries. The following preliminaries may be found in [6]. A $2 \times 2$ integral matrix $\left(a_{i j}\right)$ of the form

$$
\left(\begin{array}{cc}
2 & a_{12} \\
a_{21} & 2
\end{array}\right)
$$

where $a_{12}, a_{21}<0$ and $a_{12} a_{21}>4$ is called a rank 2 hyperbolic GCM. It gives rise to a Lie algebra $g^{\prime}$ with generators $e_{1}, e_{2}, f_{1}, f_{2}, h_{1}, h_{2}$ and relations

$$
\begin{aligned}
{\left[h_{i}, e_{j}\right] } & =a_{i j} e_{j}, \quad\left[h_{i}, f_{j}\right]=-a_{i j} f_{j}, \quad\left[h_{i}, h_{j}\right]=0, \\
{\left[e_{i}, f_{j}\right] } & =\delta_{i j} h_{j} \quad \text { for } i, j \in\{1,2\}, \text { and } \\
\left(\operatorname{ad} e_{i}\right)^{-a_{i j}+1} e_{j} & =0=\left(\operatorname{ad} f_{i}\right)^{-a_{i j}+1} f_{j} \quad \text { for } i \neq j .
\end{aligned}
$$

If $Z=Z \alpha_{1}+Z \alpha_{2}$ is a rank 2 free abelian group, one gives $g^{\prime}$ a $Z$-grading by setting $\operatorname{deg} e_{i}=\alpha_{i}, \operatorname{deg} f_{i}=-\alpha_{i}$ and $\operatorname{deg} h_{i}=0$ for $i=1,2$. If $h^{\prime}=\mathbf{R} h_{1}+\mathbf{R} h_{2}$

\footnotetext{
Received by the editors September 24, 1979; presented to the Society, January 24, 1979.

AMS (MOS) subject classifications (1970). Primary 17B65; Secondary $10 \mathrm{~A} 40$.

Key words and phrases. Lie algebras, Fibonacci numbers.

${ }^{1}$ This work was partially supported by National Science Foundation Grant MCS78-03112 while the author was at Drexel University, Philadelphia, Pennsylvania 19104.
} 
then those $\beta \in Z$ for which the degree space $g_{\beta}^{\prime}$ is nonzero are called the roots of $g^{\prime}$ with respect to $h^{\prime}$. Let $\Delta$ be the set of all roots of $g^{\prime}$. One knows that $g_{0}^{\prime}=h^{\prime}, \alpha \in \Delta$ implies $-\alpha \in \Delta$, and $m_{\alpha}^{\prime}=\operatorname{dim} g_{\alpha}^{\prime}$ is finite for all $\alpha \in \Delta$. If we define the set $\Delta^{+}$of positive roots to be $\Delta \cap\left(\mathbf{Z}^{+} \alpha_{1}+\mathbf{Z}^{+} \alpha_{2}\right)-\{0\}$ and the set $\Delta^{-}$of negative roots to be $-\Delta^{+}$then $\Delta=\Delta^{+} \cup\{0\} \cup \Delta^{-}$and $g^{\prime}$ has the root-space decomposition $g^{\prime}=$ $\Sigma_{\alpha \in \Delta^{+}} g_{\alpha}^{\prime} \oplus \Sigma_{\alpha \in \Delta^{-}} g_{\alpha}^{\prime} \oplus h^{\prime}$.

The GCM Lie algebra we want to work with is $g=g^{\prime} / r$, where $r$ is the unique maximal ideal of $g^{\prime}$. It is known that if $g_{\beta}^{\prime} \neq 0$ then $g_{\beta} \neq 0$, so the roots are unchanged. One usually just drops the prime notation used above and continues to write $e_{i}, f_{i}$ and $h_{i}$, etc., for their natural images in $g$.

Let $\mathbf{R} Z=\mathbf{R} \otimes_{\mathbf{Z}} Z$ and define the pairing $\mathbf{R} Z \times h \rightarrow \mathbf{R}$ by $\left(\alpha_{i}, h_{j}\right)=a_{j i}$. This identifies $\mathbf{R} Z$ with the dual space $h^{*}$ of $h$ and we write $\alpha_{i}\left(h_{j}\right)$ for $\left(\alpha_{i}, h_{j}\right)$. Then $g_{\beta}=\{x \in g \mid[H, x]=\beta(H) x$ for all $H \in h\}$. Let $\left\{\omega_{1}, \omega_{2}\right\} \subset \mathbf{R Z}$ be the basis dual to $\left\{h_{1}, h_{2}\right\}$. Then the weight lattice $\Omega=\mathbf{Z} \omega_{1}+\mathbf{Z} \omega_{2}$ contains the root lattice $Z$. The set of dominant weights $\Omega^{+}$is defined to be $\left\{\lambda \in \Omega \mid \lambda\left(h_{i}\right)>0\right.$ for $\left.i=1,2\right\}$.

One can define a nondegenerate symmetric bilinear form $k$ on $\mathbf{R} Z$ relative to the basis $\left\{\alpha_{1}, \alpha_{2}\right\}$ by the matrix

$$
\left(\begin{array}{cc}
2 a_{21} d & a_{12} a_{21} d \\
a_{21} a_{12} d & 2 a_{12} d
\end{array}\right) .
$$

The positive rational number $d$ is to be determined later. Note that $a_{i j}=$ $2 k\left(\alpha_{i}, \alpha_{j}\right) / k\left(\alpha_{i}, \alpha_{i}\right)$.

The Weyl group $W$ is the group of endomorphisms of $Z$ generated by $r_{1}$ and $r_{2}$ where $r_{i} \alpha_{j}=\alpha_{j}-a_{i j} \alpha_{i}$ for $i, j \in\{1,2\}$. Let $W^{+}$be the infinite cyclic subgroup of $W$ generated by $\left(r_{1} r_{2}\right)$. The only essential relations among the group generators are $r_{1}^{2}=r_{2}^{2}=1$. Every element of $W$ can be written uniquely in one of the two forms $\left(r_{1} r_{2}\right)^{i}$ or $r_{2}\left(r_{1} r_{2}\right)^{i}$ for $i \in \mathbf{Z}$. The set of roots $\Delta$ is stable under $W$ as are the dimensions of the root spaces. The set $\Delta_{R}$ of real roots is the set of $W$-conjugates of the simple roots $\alpha_{1}$ and $\alpha_{2}$. All the other roots are called imaginary and they constitute the set $\Delta_{I}$. The R-linear extensions of $r_{1}$ and $r_{2}$ to $\mathbf{R Z}$ are the simple reflections with respect to $\alpha_{1}$ and $\alpha_{2}$, respectively, and $W$ acts as isometries on $\mathbf{R Z}$ with respect to the form $k$. For $w \in W$, let $l(w)$ denote the minimal length of $w$ as a product of simple reflections and let $\operatorname{sgn}(w)=(-1)^{l(w)}$. Since $d$ is chosen positive, $k(\alpha, \alpha)<0$ for real roots $\alpha$. In [11] Moody has shown that $\Delta_{I}=\{\alpha \in Z \mid k(\alpha, \alpha)$ $>0\}$.

For each $\alpha \in \Omega$ we introduce a symbol $e(\alpha)$ and consider the set $T$ of all formal sums $\sum_{\alpha \in \Omega} x_{\alpha} e(\alpha)$ with the property that there exists a finite number of elements of $\Omega, \gamma_{1}, \gamma_{2}, \ldots, \gamma_{t}$, such that $x_{\alpha}=0$ if $\alpha \notin \cup_{j=1}^{t} \cup_{m, n>0}\left\{\gamma_{j}-m \alpha_{1}-n \alpha_{2}\right\}$. Then $T$ is an integral domain if

$$
\sum x_{\alpha} e(\alpha)+\sum y_{\alpha} e(\alpha)=\sum\left(x_{\alpha}+y_{\alpha}\right) e(\alpha)
$$

and

$$
\left(\sum x_{\alpha} e(\alpha)\right)\left(\sum y_{\beta} e(\beta)\right)=\sum_{\alpha, \beta} x_{\alpha} y_{\beta} e(\alpha+\beta)
$$

It is in $T$ that one has the character and denominator formulas. We are concerned with the latter here. Let $\rho=\omega_{1}+\omega_{2}$ and $m_{\alpha}=\operatorname{dim} g_{\alpha}$. 
TheOREM (THE WeYl-MaCDONALD-KaC DENOMINATOR FORMULA).

$$
\prod_{\alpha \in \Delta^{-}}(1-e(\alpha))^{m_{\alpha}}=\sum_{w \in W} \operatorname{sgn}(w) e(w \rho-\rho) .
$$

It is known that $\rho-w_{1} \rho=\rho-w_{2} \rho$ if and only if $w_{1}=w_{2}$, and that $\rho-w \rho=$ $\Sigma_{\alpha \in w \Delta^{-} \cap \Delta^{+}} \alpha$. Both sides of this formula can therefore be expressed as formal power series in the independent variables $u=e\left(-\alpha_{1}\right)$ and $v=e\left(-\alpha_{2}\right)$. We will show that for one algebra the exponents of $u$ and $v$ are simple linear combinations of the Fibonacci numbers.

2. The specific case of $a_{12}=a_{21}=-3$. Suppose that $a_{12}=a_{21}=-3$. Then $\alpha_{1}\left(h_{2}\right)=-3=\alpha_{2}\left(h_{1}\right)$ and so $\alpha_{1}=2 \omega_{1}-3 \omega_{2}$ and $\alpha_{2}=-3 \omega_{1}+2 \omega_{2}$. Also, $\omega_{1}=$ $-\frac{1}{5}\left(2 \alpha_{1}+3 \alpha_{2}\right)$ and $\omega_{2}=-\frac{1}{5}\left(3 \alpha_{1}+2 \alpha_{2}\right)$ so $\rho=\omega_{1}+\omega_{2}=-\alpha_{1}-\alpha_{2}$. The simple reflections satisfy $r_{1}\left(\alpha_{2}\right)=\alpha_{2}+3 \alpha_{1}$ and $r_{2}\left(\alpha_{1}\right)=\alpha_{1}+3 \alpha_{2}$. Using this and the usual root-string properties (see [11]) one can graph part of the root system for this algebra. (See Figure 1.) It may be seen that roots which are $W$-conjugate are distributed on the branches of a hyperbola. Also, the dominant chamber $\Omega^{+}$ contains exactly one representative from each $W$-conjugacy class of the negative imaginary roots $\Delta^{-}$. These representatives are the elements of the form $5 m \omega_{i}+n \rho$ for $i \in\{1,2\}, m, n>0,(m, n) \neq(0,0)$. If $n=0$ then the distinct $W$-conjugates of $5 m \omega_{i}$ are its images under $W^{+}$. If $n \neq 0$ then all the $W$-conjugates of $5 m \omega_{i}+n \rho$ are distinct. The only other negative roots are the real roots:

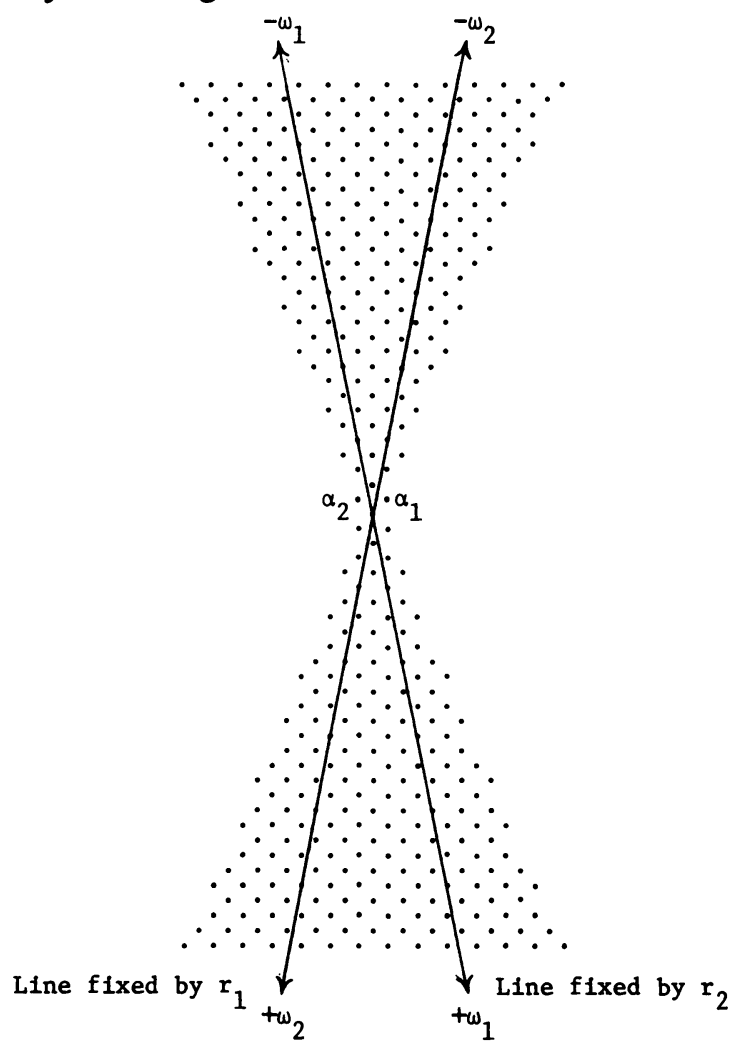

FIGURE 1: Root System 


$$
\begin{aligned}
\left(r_{1} r_{2}\right)^{i}\left(-\alpha_{1}\right) & \text { for } i>0, \\
r_{2}\left(r_{1} r_{2}\right)^{i}\left(-\alpha_{1}\right) & \text { for } i \geqslant 0, \\
\left(r_{1} r_{2}\right)^{i}\left(-\alpha_{2}\right) & \text { for } i<0, \\
r_{2}\left(r_{1} r_{2}\right)^{i}\left(-\alpha_{2}\right) & \text { for } i<0 .
\end{aligned}
$$

As Lepowsky and Moody have shown in [6], a convenient way to compute the action of $W$ is to find a basis in $R Z$ with respect to which the endomorphism $r_{1} r_{2}$ is diagonal. The eigenvalues of $r_{1} r_{2}$ are then the units $\varepsilon$ and $\varepsilon^{-1}$ in the real quadratic field $Q(\sqrt{D})$, where $D=a_{12} a_{21}\left(a_{12} a_{21}-4\right)$ and $\varepsilon=\left(\left(a_{12} a_{21}-2\right)+\sqrt{D}\right) / 2$. If $\gamma^{+}$is an eigenvector of $r_{1} r_{2}$ with eigenvalue $\varepsilon$ and $\gamma^{-}=r_{2} \gamma^{+}$then $\gamma^{-}$is an eigenvector for $r_{1} r_{2}$ with eigenvalue $\bar{\varepsilon}=\varepsilon^{-1}$ (here the "bar" denotes conjugation in the field). So with respect to the basis $\left\{\gamma^{+}, \gamma^{-}\right\}$the Weyl group elements of the form $\left(r_{1} r_{2}\right)^{i}$ (for $i \in \mathbf{Z}$ ) have matrix representations $\left(\begin{array}{l}e^{i} \\ 0 \\ e^{-i}\end{array}\right)$ and those of the form $r_{2}\left(r_{1} r_{2}\right)^{i}$ (for $i \in Z$ ) have matrix representations $\left(\left(_{e^{i}}^{e^{-i}}\right)\right.$. This is consistent with the column vector notation $\left(\begin{array}{l}x \\ y\end{array}\right)$ for $x \gamma^{+}+y \gamma^{-}$.

In the case where $a_{12}=a_{21}=-3$ we have $D=45$ and $\varepsilon=(7+3 \sqrt{5}) / 2$ which is in the quadratic field $Q(\sqrt{5})$. The algebraic integers are $\{m+n \omega \mid m, n \in$ $Z$ \}, where $\omega=(1+\sqrt{5}) / 2$ and $\omega^{4}=\varepsilon$. Note that $\bar{\omega}=(1-\sqrt{5}) / 2$ satisfies $\omega \bar{\omega}=-1$ so $\omega^{-1}=-\bar{\omega}$. The Weyl group element $\left(r_{1} r_{2}\right)^{i}$ has the matrix representation

$$
\left(\begin{array}{cc}
\omega^{4 i} & 0 \\
0 & \bar{\omega}^{4 i}
\end{array}\right)
$$

and the element $r_{2}\left(r_{1} r_{2}\right)^{i}$ has the matrix representation

$$
\left(\begin{array}{cc}
0 & \bar{\omega}^{4 i} \\
\omega^{4 i} & 0
\end{array}\right)
$$

with respect to $\gamma^{+}=(1 / 45)\left(-3 \alpha_{1}-(\bar{\varepsilon}+1) \alpha_{2}\right)=-(1 / 15)\left(\alpha_{1}+\bar{\omega}^{2} \alpha_{2}\right)$ and $\gamma^{-}=$ $(1 / 45)\left(-3 \alpha_{1}-(\varepsilon+1) \alpha_{2}\right)=-(1 / 15)\left(\alpha_{1}+\omega^{2} \alpha_{2}\right)$. (We have made the choice for the positive rational number $d=15$ which makes $k\left(\gamma^{+}, \gamma^{-}\right)=1$.) We have

$$
\begin{gathered}
\alpha_{1}=\left(\begin{array}{c}
-3(\varepsilon-1) \\
-3(\bar{\varepsilon}-1)
\end{array}\right)=\left(\begin{array}{c}
-3 \sqrt{5} \omega^{2} \\
3 \sqrt{5} \bar{\omega}^{2}
\end{array}\right), \quad \alpha_{2}=\left(\begin{array}{c}
3 \sqrt{5} \\
-3 \sqrt{5}
\end{array}\right), \\
\omega_{1}=\left(\begin{array}{l}
3 \\
3
\end{array}\right), \quad \omega_{2}=\left(\begin{array}{c}
\varepsilon+1 \\
\bar{\varepsilon}+1
\end{array}\right)=\left(\begin{array}{c}
3 \omega^{2} \\
3 \bar{\omega}^{2}
\end{array}\right) .
\end{gathered}
$$

Since $\omega$ and $\bar{\omega}$ are the roots of $x^{2}-x-1=0$ one knows that both satisfy $x^{i}=x^{i-1}+x^{i-2}$ for all $i \in \mathbf{Z}$. This allows one to write $\omega^{i}=a_{i} \omega+a_{i-1}$ where the $a_{i}$ are the Fibonacci numbers defined by the conditions $a_{0}=0, a_{1}=1, a_{i}=a_{i-1}+$ $a_{i-2}$ for all $i \in \mathbf{Z}$. One checks that $a_{-i}=(-1)^{i+1} a_{i}, \omega^{i}+\bar{\omega}^{i}=a_{i+1}+a_{i-1}, \omega^{i}-\bar{\omega}^{i}$ $=a_{i}(\omega-\bar{\omega})=a_{i} \sqrt{5}$ and $\omega^{2 i}+\bar{\omega}^{2 i}+\omega^{2 i-2}+\bar{\omega}^{2 i-2}=(\omega-\bar{\omega})\left(\omega^{2 i-1}-\bar{\omega}^{2 i-1}\right)=$ $5 a_{2 i-1}$. Let us now write down the summation side of the denominator formula. 
Since

$$
\begin{gathered}
\rho=\omega_{1}+\omega_{2}=\left(\begin{array}{c}
3 \sqrt{5} \omega \\
-3 \sqrt{5} \bar{\omega}
\end{array}\right) \\
\left(r_{1} r_{2}\right)^{i} \rho=\left(\begin{array}{c}
3 \sqrt{5} \omega^{4 i+1} \\
-3 \sqrt{5} \bar{\omega}^{4 i+1}
\end{array}\right) \text { and } r_{2}\left(r_{1} r_{2}\right)^{i} \rho=\left(\begin{array}{c}
-3 \sqrt{5} \bar{\omega}^{4 i+1} \\
3 \sqrt{5} \omega^{4 i+1}
\end{array}\right) .
\end{gathered}
$$

Using $e\left(\gamma^{+}\right)=u^{1 / 15} v^{\bar{\omega}^{2} / 15}$ and $e\left(\gamma^{-}\right)=u^{1 / 15} v^{\omega^{2} / 15}$ we see that

$$
\begin{aligned}
\sum_{w \in W} \operatorname{sgn}(w) e(w \rho)= & \sum_{i \in \mathbf{Z}}\left[u^{3 \sqrt{5}\left(\omega^{4 i+1}-\bar{\omega}^{4 i+1}\right) / 15} v^{3 \sqrt{5}\left(\omega^{4 i-1}-\bar{\omega}^{4 i-1}\right) / 15}\right. \\
& \left.-u^{3 \sqrt{5}\left(\omega^{4 i+1}-\bar{\omega}^{4+1}\right) / 15} v^{3 \sqrt{5}\left(\omega^{4 i+3}-\bar{\omega}^{4 i+3}\right) / 15}\right] \\
= & \sum_{i \in \mathbf{Z}}\left[u^{a_{4 i+}+v^{a_{4 i-1}}-u^{\left.a_{4 i+}+v^{a_{4 i+3}}\right]}}\right. \\
= & \sum_{j \in \mathbf{Z}}(-1)^{j} u^{a_{2 j+} v^{a_{2 j-1}} .}
\end{aligned}
$$

So $\sum_{w \in W} \operatorname{sgn}(w) e(w \rho-\rho)=u^{-1} v^{-1} \sum_{j \in \mathbf{Z}}(-1)^{j} u^{a_{2 j+}+v^{a_{2 j-1}}}$ is the summation side of the denominator formula for this algebra.

In order to write down the product side of the denominator formula we must list all the negative roots, each exactly once. We have already done this for the negative real roots. The negative imaginary roots are as follows:

Case 1. If $m \geqslant 0$ and $n>0$ then all $W$-conjugates of $5 m \omega_{1}+n \rho$ are distinct.

Case 2. If $m>0$ and $n>0$ then all $W$-conjugates of $5 m \omega_{2}+n \rho$ are distinct and different from all those of Case 1 .

Case 3. If $n=0$ and $m>0$ then all $W^{+}$-conjugates of $5 m \omega_{1}$ are distinct negative roots not yet listed.

Case 4. If $n=0$ and $m>0$ then all $W^{+}$-conjugates of $5 m \omega_{2}$ are distinct negative roots not yet listed.

In each of the above cases we shall use the notation $C(m, n)$ for the root multiplicity of $5 m \omega_{1}+n \rho$, which, by the special symmetry of this algebra $\left(a_{12}=\right.$ $a_{21}=-3$ ), is the same as the multiplicity of $5 m \omega_{2}+n \rho$. The multiplicity of real roots is 1 .

Case $1(m \geqslant 0, n>0)$.

$$
W\left(5 m \omega_{1}+n \rho\right)=\left\{\left(\begin{array}{c}
\left(15 m+3 n\left(\omega^{2}+1\right)\right) \omega^{4 i} \\
\left(15 m+3 n\left(\bar{\omega}^{2}+1\right)\right) \bar{\omega}^{4 i}
\end{array}\right),\left(\begin{array}{c}
\left(15 m+3 n\left(\bar{\omega}^{2}+1\right)\right) \bar{\omega}^{4 i} \\
\left(15 m+3 n\left(\omega^{2}+1\right)\right) \omega^{4 i}
\end{array}\right) \mid i \in \mathbf{Z}\right\}
$$

Case 2. $(m>0, n>0)$.

$$
\begin{aligned}
W\left(5 m \omega_{2}+n \rho\right)=\left\{\left(\begin{array}{l}
\left(15 m \omega^{2}+3 n\left(\omega^{2}+1\right)\right) \omega^{4 i} \\
\left(15 m \bar{\omega}^{2}+3 n\left(\bar{\omega}^{2}+1\right)\right) \bar{\omega}^{4 i}
\end{array}\right)\right. \\
\left.\left(\begin{array}{c}
\left(15 m \bar{\omega}^{2}+3 n\left(\bar{\omega}^{2}+1\right)\right) \bar{\omega}^{4 i} \\
\left(15 m \omega^{2}+3 n\left(\omega^{2}+1\right)\right) \omega^{4 i}
\end{array}\right) \mid i \in \mathbf{Z}\right\} .
\end{aligned}
$$


Case 3. $(n=0, m>0)$.

$$
W\left(5 m \omega_{1}\right)=\left\{\left(\begin{array}{c}
15 m \omega^{4 i} \\
15 m \bar{\omega}^{4 i}
\end{array}\right) \mid i \in \mathbf{Z}\right\} .
$$

Case 4. $(n=0, m>0)$.

$$
W\left(5 m \omega_{2}\right)=\left\{\left(\begin{array}{c}
15 m \omega^{4 i+2} \\
15 m \bar{\omega}^{4 i+2}
\end{array}\right) \mid i \in \mathbf{Z}\right\} .
$$

Case 5. The negative real roots are

$$
\begin{aligned}
\left\{\left(\begin{array}{c}
3 \sqrt{5} \omega^{4 i+2} \\
-3 \sqrt{5} \bar{\omega}^{4 i+2}
\end{array}\right) \mid i \geqslant 0\right\}, & \left\{\left(\begin{array}{c}
-3 \sqrt{5} \bar{\omega}^{4 i+2} \\
3 \sqrt{5} \omega^{4 i+2}
\end{array}\right) \mid i>0\right\}, \\
\left\{\left(\begin{array}{c}
-3 \sqrt{5} \omega^{4 i} \\
3 \sqrt{5} \bar{\omega}^{4 i}
\end{array}\right) \mid i \leqslant 0\right\}, & \left\{\left(\begin{array}{c}
3 \sqrt{5} \bar{\omega}^{4 i} \\
-3 \sqrt{5} \omega^{4 i}
\end{array}\right) \mid i<0\right\} .
\end{aligned}
$$

If we change $i$ to $-i$ in the last two sets they combine with the first two, giving

$$
\left\{\left(\begin{array}{c}
-3 \sqrt{5} \bar{\omega}^{2 i} \\
3 \sqrt{5} \omega^{2 i}
\end{array}\right) \mid i \geqslant 0\right\}, \quad\left\{\left(\begin{array}{c}
3 \sqrt{5} \omega^{2 i} \\
-3 \sqrt{5} \bar{\omega}^{2 i}
\end{array}\right) \mid i>0\right\} .
$$

Cases 3 and 4 may be combined to give

$$
\left\{\left(\begin{array}{c}
15 m \omega^{2 i} \\
15 m \bar{\omega}^{2 i}
\end{array}\right) \mid i \in \mathbf{Z}, m>0\right\}
$$

which, after some minor transformations, combines with Cases 1 and 2 to give all the negative imaginary roots;

$$
\begin{aligned}
& \left\{\left(\begin{array}{c}
\left(15 m+3 n\left(\bar{\omega}^{2}+1\right)\right) \bar{\omega}^{2 i} \\
\left(15 m+3 n\left(\omega^{2}+1\right)\right) \omega^{2 i}
\end{array}\right) \mid i \in \mathbf{Z}, m>0, n>0\right\}, \\
& \left\{\left(\begin{array}{c}
\left(15 m+3 n\left(\omega^{2}+1\right)\right) \omega^{2 i} \\
\left(15 m+3 n\left(\bar{\omega}^{2}+1\right)\right) \bar{\omega}^{2 i}
\end{array}\right) \mid i \in \mathbf{Z}, m>0, n>0\right\} .
\end{aligned}
$$

If we use this information to write the product side of the denominator formula in terms of $u$ and $v$, considerable simplification occurs. One finds that

$$
\begin{aligned}
\prod_{\alpha \in \Delta^{-}}(1-e(\alpha))^{m_{\alpha}}= & \prod_{\substack{i \in \mathbf{Z} \\
m>0 \\
n>0}}\left(1-u^{(m+n) a_{2 i+1}+m a_{2 i-1}} v^{(m+n) a_{2 i+3}+m a_{2 i+1}}\right)^{C(m, n)} \\
& \cdot \prod_{\substack{i \in \mathbf{Z} \\
m>0 \\
n>0}}\left(1-u^{(m+n) a_{2 i+1}+m a_{2 i-1}} v^{(m+n) a_{2 i-1}+m a_{2 i-3}}\right)^{C(m, n)} \\
& \cdot \prod_{i>0}\left\{\left(1-u^{a_{2 i} v^{a_{2 i+2}}}\right)\left(1-u^{\left.a_{2 i+2} v^{a_{2 i}}\right)}\right\} .\right.
\end{aligned}
$$

Replace $i$ by $-i$ in the first of these products and let $p=m$ and $q=m+n$. A simple calculation shows that $5 p \omega_{i}+(q-p) \rho$ and $5 q \omega_{i}+(p-p) \rho$ are $W$-conjugate, so that $C(p, q-p)=C(q, p-q)$. This allows one further simplification since $\{p>q>0\} \cup\{q>p>0\}=\{p>0, q>1\}$. 
TheOREM. The Weyl-Macdonald-Kac denominator formula for the GCM Lie algebra whose matrix is $\left(\begin{array}{cc}2 & 2^{-3}\end{array}\right)$ may be written in the following form:

$$
\begin{gathered}
\prod_{\substack{i \in \mathrm{Z} \\
p>0 \\
q>1}}\left(1-u^{p a_{2 i-1}+q a_{2 i+w}} \boldsymbol{w}^{q a_{2 i-1}+p a_{2 i-3}}\right)^{C(p, q-p)} \cdot \prod_{i>0}\left\{\left(1-u^{\left.\left.a_{2 i} v^{a_{2 i+2}}\right)\left(1-u^{a_{2 i+2}} v^{a_{2 i}}\right)\right\}}\right.\right. \\
=u^{-1} v^{-1} \sum_{j \in \mathrm{Z}}(-1)^{j} u^{a_{2 j+w} v^{a_{2 j-1}}}
\end{gathered}
$$

where $C(m, n)$ is the root multiplicity of $5 m \omega_{i}+n \rho$ for $i=1,2$ and $a_{i}$ is the ith Fibonacci number for all $i \in \mathbf{Z}$.

3. Comments. After "principal" specialization $(u=v=x$, see $[5, \S 17])$ of the two-variable power series identity above one obtains a one-variable identity which may be used to compute recursively the root multiplicities $C(m, n)$. Also see [1].

While this computation is only for one specific algebra it is clear that similar computations are possible for all of the rank 2 hyperbolic GCM Lie algebras. In those cases a role is played by generalized Fibonacci numbers.

\section{REFERENCES}

1. S. Berman and R. V. Moody, Lie algebra multiplicities, Proc. Amer. Math. Soc. 76 (1979), 223-228.

2. V. G. Kac, Simple irreducible graded Lie algebras of finite growth, Izv. Akad. Nauk SSSR Ser. Mat. 32 (1968), 1323-1367; English transl.,Math USSR-Izv. 2 (1968), 1271-1311.

3. , Infinite-dimensional Lie algebras and Dedekind's $\eta$-function, Funkcional Anal. i Priložen. 8 (1974), 77-78; English transl., Functional Anal. Appl. 8 (1974), 68-70.

4. J. Lepowsky, Lie algebras and combinatorics, Proc. Internat. Congr. Math. (Helsinki, 1978) (to appear).

5. __ Generalized Verma modules, loop space cohomology and Macdonald-type identities, Ann. Sci. Ecole Norm. Sup. 12 (1979), 169-234.

6. J. Lepowsky and R. V. Moody, Hyperbolic Lie algebras and quasi-regular cusps on Hilbert modular surfaces, Math. Ann. 245 (1979), 63-88.

7. I. G. Macdonald, Affine root systems and Dedekind's $\eta$-function, Invent. Math. 15 (1972), 91-143.

8. R. V. Moody, A new class of Lie algebras, J. Algebra 10 (1968), 210-230.

9. ___ Euclidean Lie algebras, Canad. J. Math. 21 (1969), 1432-1454.

10. _ Macdonald identities and Euclidean Lie algebras, Proc. Amer. Math. Soc. 48 (1975), 43-52.

11. , Root systems of hyperbolic type, Advances in Math. 33 (1979), 144-160.

Department of Mathematics, State University of New York, Bngghamton, New York 13901 